\title{
Longitudinal acceptance measurement at an electron storage ring
}

\author{
Peter Kuske and Ji Li॰ \\ Helmholtz-Zentrum Berlin für Materialien und Energie GmbH, 14109 Berlin, Germany \\ Masamitsu Aiba® \\ Paul Scherrer Institut, Villigen CH-5232, Switzerland
}

(Received 5 December 2019; accepted 18 February 2020; published 4 March 2020)

\begin{abstract}
The longitudinal acceptance of the BESSY II storage ring has been measured. To our knowledge, such a measurement has never been performed in electron storage rings. The study is also motivated by the fact that for future diffraction limited light sources a couple of longitudinal injection schemes were proposed recently. In these injection schemes, the energy and/or timing of the injection beam is shifted, resulting in a large synchrotron oscillation amplitude of the injected beam. It is, therefore, of importance to reveal whether we can evaluate the longitudinal acceptance precisely when we design a storage ring with a longitudinal injection scheme. The experimental results showed a good agreement to the acceptance evaluated through the accelerator model, including the special feature arising from the synchrotron radiation. We also found that, as a by-product, the bunch length of the injected beam can be obtained from the acceptance measurement data. The result is in good agreement with the design value of the injector booster synchrotron.
\end{abstract}

DOI: 10.1103/PhysRevAccelBeams.23.030701

\section{INTRODUCTION}

Modern high-performance accelerators are designed, constructed and brought into operation based on a precise accelerator model. The electromagnetic fields of all components are accurately computed at the design phase, measured for most components, and finely tuned based on beam measurements. After all these efforts, the design performance is achieved eventually.

This may be also true for next-generation light-source storage rings. They are based on multibend achromat lattices to lower the natural beam emittance through strong focusing and low dispersion function. Consequently, quite strong chromaticity correction sextupoles are required, and the dynamic aperture tends to be insufficient for the conventional beam injection scheme. Since the problem is highly nonlinear, the evaluation of the dynamic aperture is normally performed through numerical tracking including the field errors and the misalignments of the accelerator components, i.e., through a complete accelerator model.

Most light sources are operated in the so-called top-up mode [1], where electrons are frequently injected atop of the stored beam, compensating for the lost particles and

Published by the American Physical Society under the terms of the Creative Commons Attribution 4.0 International license. Further distribution of this work must maintain attribution to the author(s) and the published article's title, journal citation, and DOI. keeping the storage ring beam current essentially constant. The top-up operation mode maximizes the performance of a light source, i.e., the photon beam flux is kept at the maximum value with the constant electron beam current. It is important also for the beam stability to keep the electron beam current constant such that the heat load due to the synchrotron radiation to the accelerator component is constant as well.

For the accumulation of beam current in the top-up mode, the injected beam has to be separated from the stored beam at least in one of the three planes (horizontal, vertical or longitudinal) at the time of injection according to Liouville's theorem. In the conventional injection scheme, the injected beam is placed transversely off axis, and thus the dynamic aperture should be large enough to accept the off-centered injected beam. This is one of the main challenges for next-generation light sources due to the strong nonlinearity in the multibend achromat lattice.

Recently, longitudinal injection schemes have been proposed for future light sources [2-6] to enable beam injection into a small transverse aperture ring using the longitudinal acceptance instead of the transverse one. The aforementioned dynamic aperture problem is not solved but then avoided. In these injection schemes, the energy and/or timing of the injection beam is shifted, resulting in a large synchrotron oscillation amplitude of the injected beam, while the injection beam is placed on axis transversely. Depending on the initial condition, the injected beam particles may or may not be captured by the rf bucket. 
The goal of this study is to reveal whether the longitudinal acceptance of the ring is consistent with a prediction from the accelerator model. We present our longitudinal acceptance measurement at BESSY II [7]. The longitudinal acceptance of the storage ring has been clearly visualized through the measurement, and to our knowledge it is the first time that such a measurement has been performed in the electron storage ring.

The paper is organized as follows. In Sec. II, longitudinal beam dynamics in electron storage rings is revisited. In Sec. III, we introduce the BESSY II accelerator complex. Afterwards, in Sec. IV, we describe how the measurement was set up and performed, and present the results obtained. We discuss the result through a comparison with simulation in Sec. V. In addition, we evaluate the bunch length of the injected beam using the measured data. Finally we draw our conclusions in Sec. VI.

\section{LONGITUDINAL BEAM DYNAMICS IN ELECTRON STORAGE RINGS}

The longitudinal beam dynamics is briefly reviewed in this section, including synchrotron radiation and radiation damping intrinsic to the electron storage ring. We consider here only the longitudinal motion of single particles but important parameters, such as the energy loss per turn and the momentum compaction factor, are obtained from the full accelerator model. For information, the accelerator code ELEGANT [8] is used to model the BESSY II ring.

The longitudinal motion can be described with the following equations of motion, assuming a relativistic beam and a linear approximation of the momentum compaction factor:

$$
\frac{d z}{d t}=-c \alpha \delta
$$

and

$$
\frac{d \delta}{d t}=\frac{e V-U}{E_{0} T_{0}},
$$

where $z$ is the longitudinal coordinate with respect to the reference synchronous particle, $c$ is the speed of light, $\alpha$ is the momentum compaction factor of the storage ring, $\delta$ is the relative energy deviation, $e$ is the electron charge, $E_{0}$ is the nominal beam energy, $T_{0}$ is the revolution period, and $U$ is the energy loss per turn due to synchrotron radiation. $V$ is the rf voltage, which is represented as

$$
V(z)=V_{0} \sin \left(2 \pi f_{\mathrm{rf}} \frac{z}{c}+\psi_{s}\right)
$$

where $\psi_{s}$ is the synchronous phase. Time, $t$, is used as an independent variable. In Eq. (1), the relative momentum deviation is approximated by the relative energy deviation, $\delta$, for relativistic beams.

The rf frequency, $f_{\text {rf }}$, is normally a high multiple of the revolution frequency, $f_{0}$, that is,

$$
f_{\text {rf }}=h f_{0},
$$

where $h$ is the so-called harmonic number. The energy loss per turn due to synchrotron radiation, $U$, in a linear approximation is [9]

$$
U(\delta) \sim U_{0}\left(1+J_{L} \delta\right)
$$

where $U_{0}$ is the energy loss per turn for the nominal beam energy, and $J_{L}$ is the longitudinal damping partition. For $|\delta|<0.05$, which is the range that concerns us here, the linear approximation is fully justified.

The energy dependence of the radiation loss is the origin of radiation damping. In contrast, if we assume that the energy loss per turn is constant over the energy deviation, a "static" rf bucket can be defined similarly to the moving (or accelerating) bucket in a synchrotron. The synchronous phase, the bucket height, and the synchrotron tune are then given by

$$
\begin{gathered}
\psi_{s}=\pi-\arcsin \left(\frac{U_{0}}{e V_{0}}\right), \\
\delta_{B}=\sqrt{\frac{2 e V_{0}}{\pi E_{0} h \alpha}\left|\cos \psi_{s}-\frac{\pi-2 \psi_{s}}{2} \sin \psi_{s}\right|},
\end{gathered}
$$

and

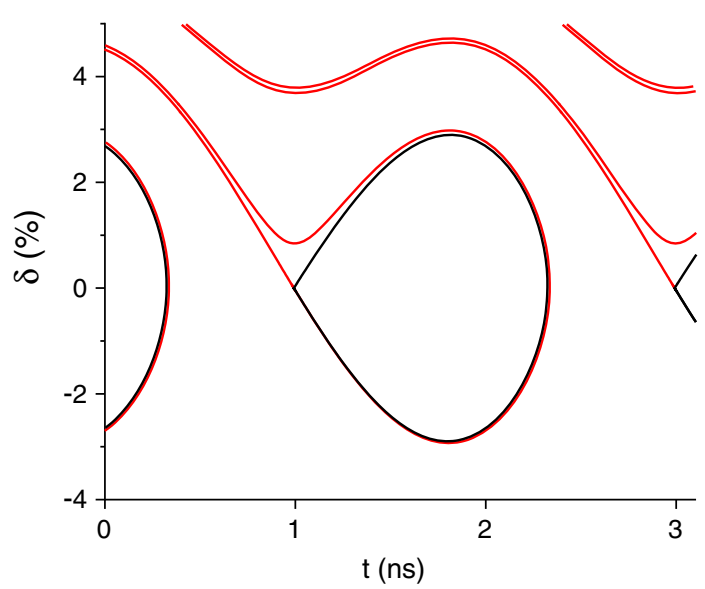

FIG. 1. Theoretical longitudinal acceptance for BESSY II storage ring parameters. A linear approximation is applied to the momentum compaction factor. The rf voltage is set to 1.12 MV. Red lines indicate the acceptance including synchrotron radiation damping while the static bucket is indicated by black lines. 
TABLE I. BESSY II storage ring and booster parameters. The energy loss varies, depending on whether the 7-Tesla superconducting wiggler and wave length shifters are turned on. The rf voltage is varied depending on operation conditions.

\begin{tabular}{lc}
\hline \hline Parameters & Values \\
\hline Circumference & $240 \mathrm{~m}$ \\
Number of cells & $16(\mathrm{DBA})$ \\
Beam energy & $1.7 \mathrm{GeV}$ \\
Radiation energy loss & $180-320 \mathrm{keV} /$ turn \\
Momentum compaction & $7.0-7.3 \times 10^{-4}$ \\
rf frequency & $500 \mathrm{MHz}$ \\
Nominal rf voltage & $1.12 \mathrm{MV}$ \\
Booster bunch length & $\sim 55 \mathrm{ps}$ \\
Booster bunch energy spread & $5.7 \times 10^{-4}$ \\
Booster beam emittance & $60 \mathrm{~nm}$ \\
\hline \hline
\end{tabular}

$$
Q_{s}=\sqrt{\frac{h \alpha e V_{0}}{2 \pi E_{0}}\left|\cos \psi_{s}\right|},
$$

respectively. See e.g., [10] for the derivation of these equations.

Figure 1 shows the longitudinal acceptance, which is computed through these equations, for the parameters of the BESSY II storage ring (see Table I in Sec. III) with an rf voltage of 1.12 MV. The computation is a simple onedimensional tracking, where the equations of motion [Eqs. (1) and (2)] are numerically integrated turn by turn.

One sees in Fig. 1 that the acceptance phase-space plot has the shape of a "golf club" with its "shaft" extending toward the neighboring bucket. In principle, the injected beam can be placed anywhere within the acceptance, even on the shaft if the longitudinal emittance is small enough to fit.

It is known that such a golf-club shape appears in the longitudinal phase space of low-energy proton linacs or synchrotrons when the rf acceleration is taken into account (see, e.g., [11]), where the parameters that appear in the above equations are varying as a function of time. In electron storage rings the parameters are usually assumed to be fixed. However, a similar effect originates from the energy- and thus time-dependent synchrotron radiation loss and the energy recovery from the rf field. The former makes the system nonconservative and leads to radiation damping.

\section{BESSY II ACCELERATOR COMPLEX}

BESSY II is a third-generation light source, providing photon beams to many beamlines for users. The parameters relevant to this study are listed in Table I, and the ideal optical functions of the storage ring are shown in Fig. 2. During user operation, a modified optics is applied, which breaks the 8 -fold symmetry and leads to a slightly smaller momentum compaction factor, $7.0 \times 10^{-4}$. The damping

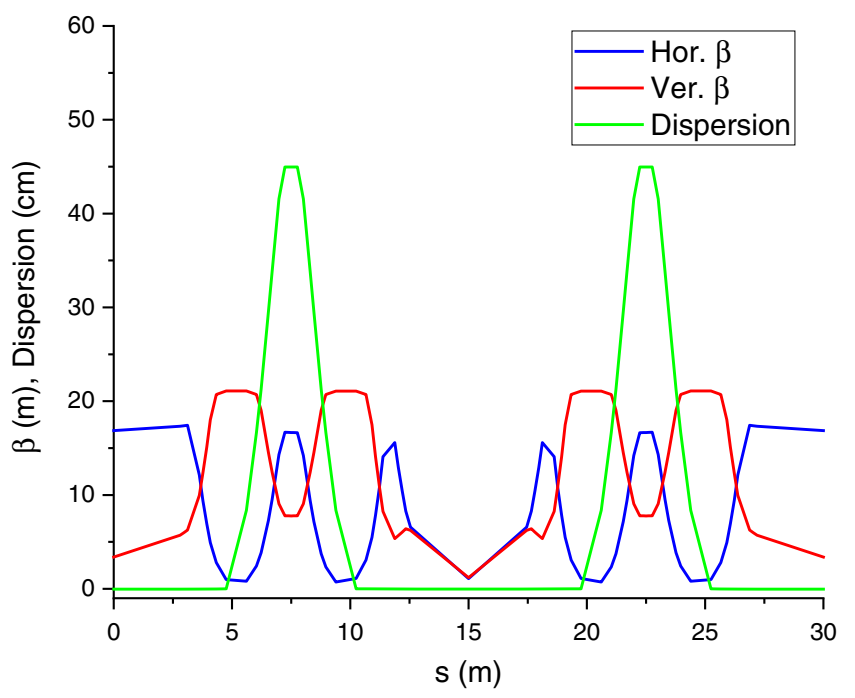

FIG. 2. Optical functions of the BESSY II storage ring (one octant). The arc is a double bend achromat (DBA) and identical for all 16 cells while there are two types of straight sections, i.e., high and low beta sections with quadrupole doublets and triplets, respectively.

partition is not changed, since the dipole magnets have no transverse gradient.

The injector consists of an electron gun, a linac, and a booster synchrotron. For the longitudinal acceptance measurement, the injection energy and timing have to be changed. The energy of the injected beam is varied by changing the extraction time in the fast cycling booster with power supplies based on White-circuits which lead to a sinusoidal energy variation at $10 \mathrm{~Hz}$. The transfer line between booster and storage ring has to be adjusted according to the chosen extraction energy. The injection timing is varied with the help of a remotely controlled motorized rf trombone allowing for a phase shift of $2.1 \mathrm{~ns}$ between booster synchrotron and storage ring and covers more than one $500 \mathrm{MHz}$ rf period. The beam injection into the storage ring is based on the conventional scheme, i.e., with an injection septum and a kicker bump. The latter consists of four dipole kickers, and the injection septum is installed between the second and third kickers [12].

A variety of beam diagnostics are installed: beam position monitors, current transfers, streak camera, diagnostics kicker, etc. This equipment is used to enable a stable operation as well as complex beam measurements in the machine development shifts.

\section{ACCEPTANCE MEASUREMENT}

The longitudinal acceptance is obtained by measuring the injection efficiency for various injection timings and injection beam energies, i.e., a two-dimensional scan in longitudinal phase space. This section describes how we performed the measurement and obtained our results. 


\section{A. Measurement setup}

We used the machine as it was for user operation, i.e., with the modified optics. The momentum compaction factor was therefore $7.0 \times 10^{-4}$ during the measurement. Prior to the acceptance measurement, the sextupole setting was optimized and confirmed to maximize the transverse off-energy acceptance. This is important since the injection beam particle can otherwise be lost transversely even when the synchrotron oscillation with large amplitude is stable.

We used a single kicker on-axis injection scheme for the experiments. Luckily, the horizontal diagnostics kicker installed in the ring has the right horizontal phase advance to accomplish this; however, the kicker is located in a straight section 14 double-bend cells downstream of the injection point. This is a long way for the injected beam injected off-energy. When the energy offset was very large, the second half of the kicker bump had to be turned on in order to let the injected beam reach the kicker, which then puts the beam transversely on axis. Accumulation (or topup injection) is impossible in this swap-out configuration. Whenever the kicker is fired and beam is injected, the beam previously captured in the storage ring is kicked out and aborted. This is very convenient for the injection efficiency measurement as discussed in the following section.

A beam position monitor operating in turn-by-turn mode was used to adjust the kicker strength: the betatron oscillation of the injection beam was clearly observed and sufficiently minimized.

\section{B. Injection efficiency measurement}

The injection efficiency is measured using two current transformers (CTs), one installed in the booster, the other in the storage ring. For the latter CT, the beam current is measured during one second after the injection. This is approximately equivalent to count the number of electrons captured into the rf bucket from the present injection beam since the electrons from the previous injection is dumped as mentioned above. The injection efficiency is computed by comparing the beam currents found from the two CTs.

The fluctuation of the booster extraction current does not influence the measurement, since the number of electrons captured by the storage ring is always normalized to the reading of the booster CT. As we will show shortly, the injection efficiency within the rf bucket can be close to $100 \%$, excluding significant beam loss in the booster-tostorage-ring transport line. The transverse acceptance of the storage ring is large enough to accept the booster beam at least for reasonable energy offsets (see Fig. 5 in Sec. V). As long as the beam loss is due to the longitudinal motion in the storage ring, the injection efficiency measurement is equivalent to the longitudinal acceptance measurement.

An application software reads out the beam current from the two CTs, calculates the injection efficiency, and continuously writes it into an EPICS channel. The beam current measured by the CTs may include an offset, and it is determined prior to each timing scan and properly subtracted by the script. This is important because typical currents to be measured are less than $1 \mathrm{~mA}$. Another application software, one of the standard tools available in the BESSY control room, runs in parallel to vary the injection timing. The injection efficiency and the corresponding injection timing are recorded through the EPICS standard strip-chart application. With these applications,
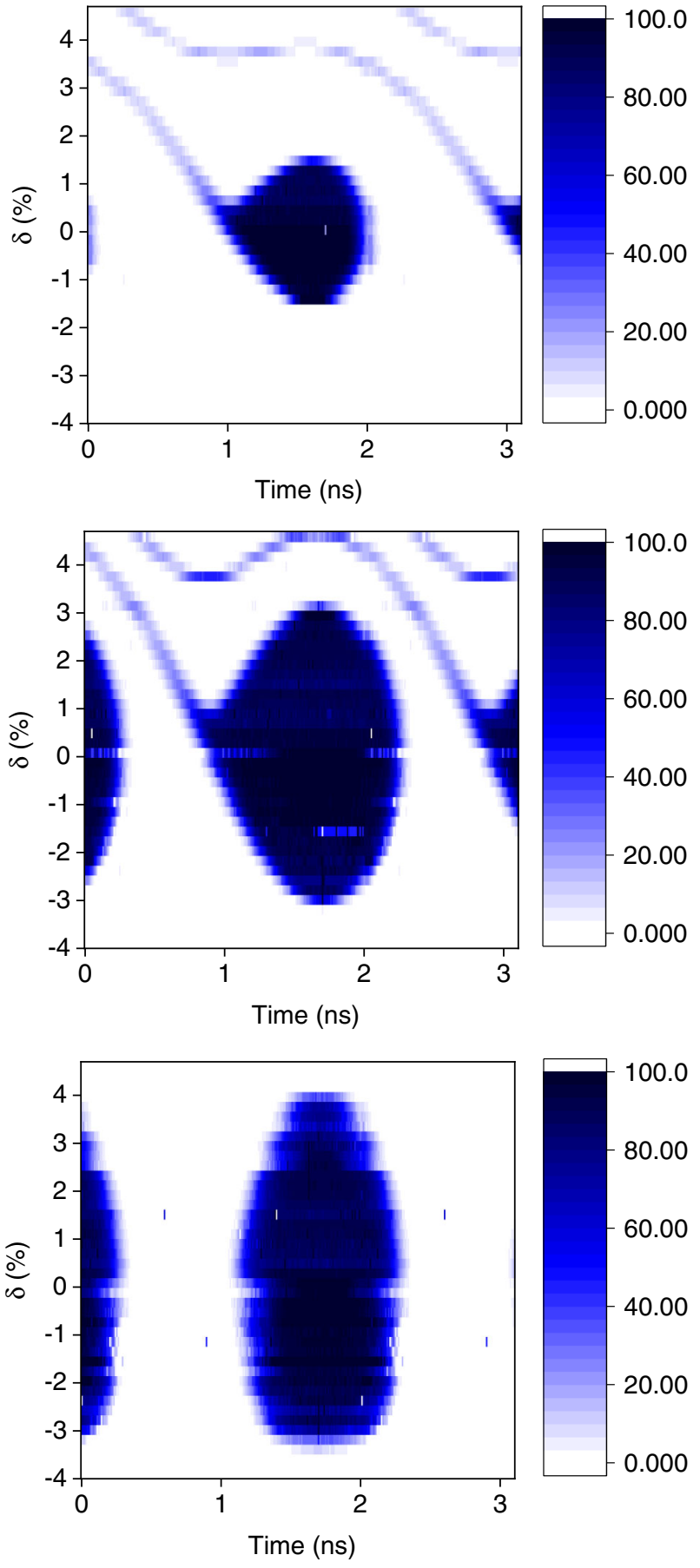

FIG. 3. Measured longitudinal acceptance. The rf voltage was set to 0.6 MV (top), 1.12 MV (middle), and 2.0 MV (bottom). 
the measurement is semiautomated. The scan of the injection timing is then repeated for various injection beam energies to complete the scan over the longitudinal phase space.

The entire procedure was repeated to measure the longitudinal acceptance for $\mathrm{rf}$ voltages of $0.6,1.12$ and 2.0 MV. It is noted that the third-harmonic passive cavity was not excited during the measurement because of the very small beam currents below $1 \mathrm{~mA}$.

\section{Results}

The measurement results for various rf voltages are shown in Fig. 3. The measured injection efficiencies are summarized in a two-dimensional color plot, which visualizes the longitudinal acceptance clearly. The step sizes of the two-dimensional scan were $0.01 \mathrm{~ns}$ in time and $0.2 \%$ in energy-offset.

\section{DISCUSSION}

For a better understanding of the measurement results presented in the previous section, they are compared to the simulated ones as shown in Fig. 4. The simulation is a simple one-dimensional tracking, using Eqs. (1) and (2), as is done in Fig. 1. The comparison is performed in two ways:
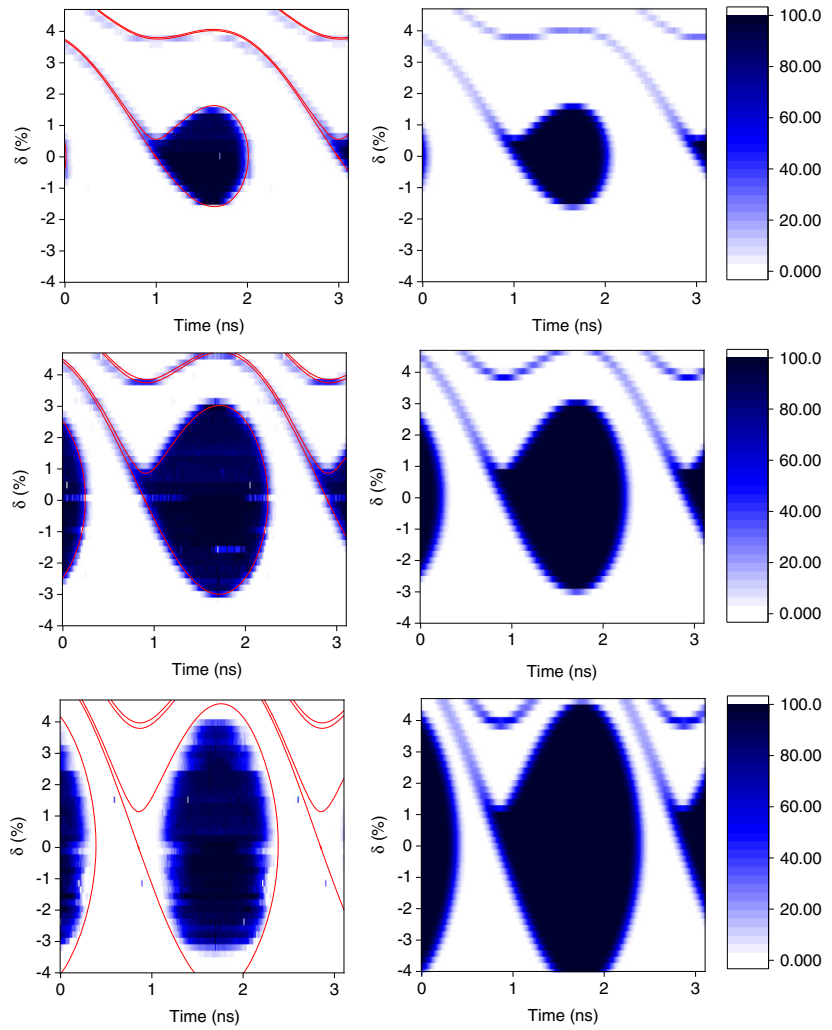

FIG. 4. Measurement data compared with simulated acceptance (left) and modeled injection efficiency (right). The rf voltage was set to 0.6 (top), 1.12 (middle), and 2.0 MV (bottom). See text for details. the simulated longitudinal acceptance in red is overlaid (left column), and the injection efficiency is computed assuming that the injected beam profile, or equivalently the booster beam profile, is Gaussian with the design bunch length of 55 ps (right column). The computation of the injection efficiency is simplified as follows. Once the longitudinal acceptance is computed, we can immediately find if a particle will be captured or not for any initial condition by definition of the acceptance. A Gaussian bunch of many particles is generated with random numbers and located at various initial energies and timings in longitudinal phase space. The injection efficiency is then computed by counting the number of particles that are within the acceptance. For a more accurate estimation the convolution of the Gaussian probability function with the acceptance should be calculated.

At the low and middle voltages (0.6 MV and 1.12 MV), the golf-club acceptance was clearly observed both in measurement and simulation. The injection efficiencies are, however, rather low at the shaft. This is attributed to the fact that the booster bunch is too long to fit the shaft. Indeed, the simulation confirms these low injection efficiencies. Overall, the agreement between measurement and simulation is quite good. The energy setting of the injection beam includes a systematic error of $0.1-0.2 \%$. This may explain the small difference at large positive $\delta$.

At the high voltage $(2.0 \mathrm{MV})$, the acceptance is a simple elliptic shape in the measurement and is not consistent with the simulation. We believe that the off-energy transverse aperture was not enough for a large negative energy deviation. With the modified optics, the theoretical transverse aperture diminishes at around $\delta=-4.7 \%$ (Fig. 5). It can be worse in the machine with imperfections such as

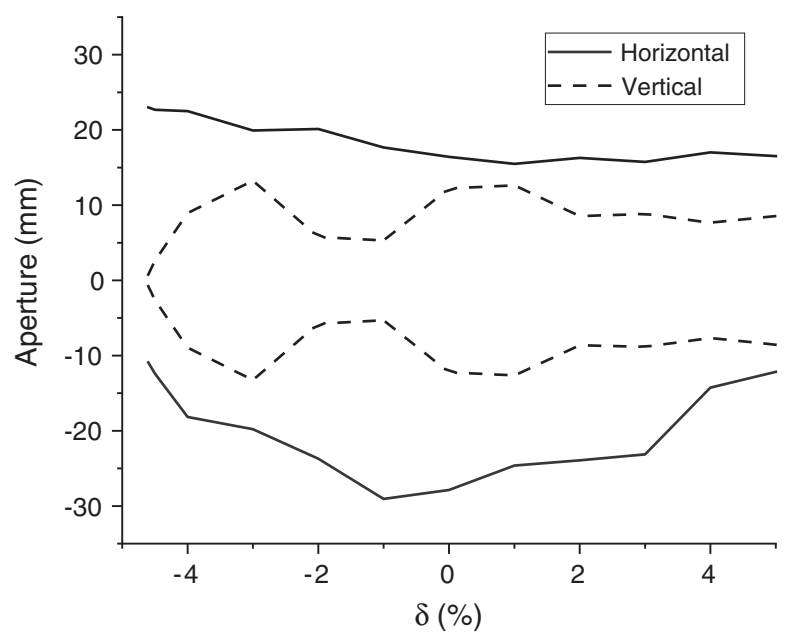

FIG. 5. Dynamic aperture of the modified optics as a function of energy offset. The beta functions are $16.5 \mathrm{~m}$ in the horizontal plane and $3.7 \mathrm{~m}$ in the vertical plane at the longitudinal location, where the aperture is computed. No machine imperfections are included. The number of turns in the tracking (elegant) is 1000 , and the rf cavities are turned off. 
misalignments and magnetic field errors even though the sextupole setting was optimized before the experiment. Such a limitation explains the observed difference. Upon a closer look, there is an asymmetry in the experimental result with respect to the energy, which is beyond the aforementioned energy setting error. Beam can be injected up to $+4 \%$ energy offset, however, for negative offset the limit is around $-3 \%$. This indicates that the longitudinal motion is stable even for $-4 \%$ negative energy offset, because the injected beam with $+4 \%$ offset reaches $-4 \%$ offset after half a synchrotron oscillation period. The injection efficiency is sensitive to transverse injection errors for rather large energy deviations where the dynamic aperture is not enough. The on-axis injection tuning might not be as precise as required for the injected beam with negative offset below $-3 \%$.

With the injection efficiency data the bunch length of the booster beam can be estimated. A part of the data obtained at the nominal beam energy is extracted and plotted in Fig. 6. Assuming that the booster bunch profile is Gaussian, an error function is fitted to the data. The bunch length from this analysis is $54 \mathrm{ps}$, which is very close to the design value of $55 \mathrm{ps}$. This justifies the assumption made for the above comparison (Fig. 4, right column).

One of the important parameters for the longitudinal particle motion is the momentum compaction factor $\alpha$. We examined the robustness of $\alpha$ against machine imperfections. Since $\alpha$ is determined by the optical function, namely the dispersion function over the dipole magnets, $\alpha$ of the BESSY II lattice is computed with quadrupole magnetic field errors that distort the storage ring optics (Fig. 7).

The momentum compaction factor is quite robust against machine imperfection. The same computation was also performed for a seven-bend achromat lattice, and a similar

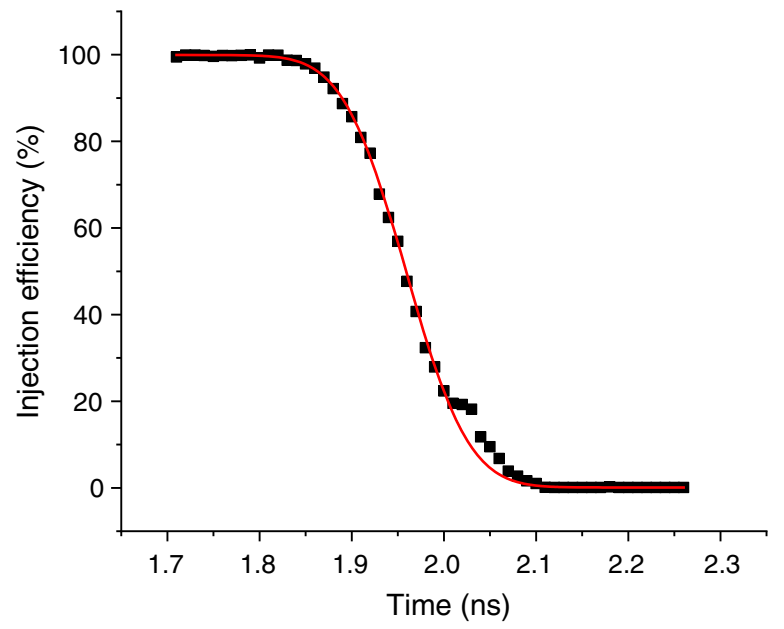

FIG. 6. Booster beam bunch length estimation using a part of the injection efficiency measurement data. The data plotted is taken from the measurement with the rf voltage of $0.6 \mathrm{MV}$ at the nominal energy. An error function is fitted (red line) to this part of the data (black points), resulting in a bunch length of 54 ps.

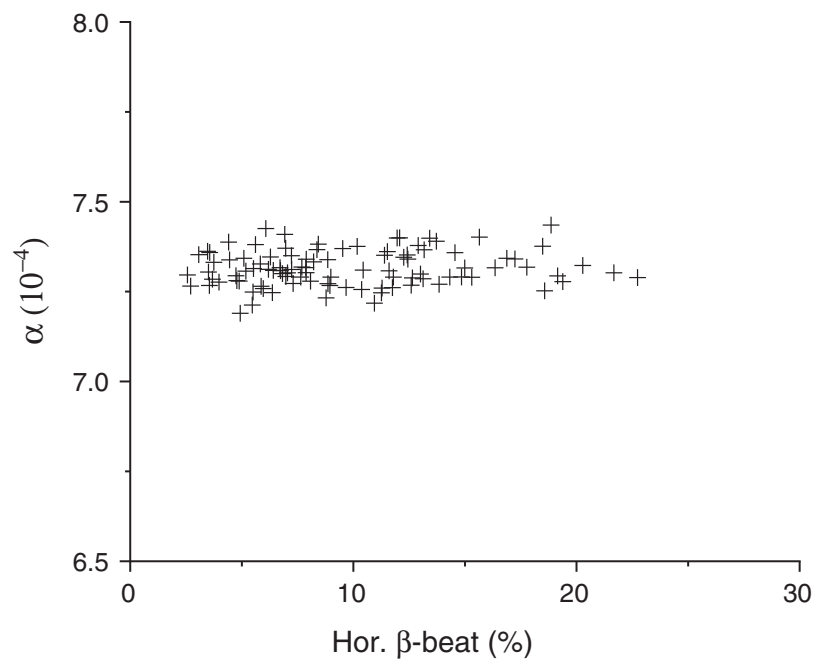

FIG. 7. Momentum compaction factor, $\alpha$, as a function of horizontal $\beta$-beat, representing the optical error. The ideal lattice (Fig. 2) is examined here. The optical error is introduced through quadrupole field errors generated with random numbers.

result was obtained. This robustness appears to be a gratifying feature common at least to achromat lattices. In addition, the linear lattice functions, including the dispersion function, of future multibend low emittance rings need to be carefully corrected in order to reach the required transverse dynamic apertures to accumulate beam even with swap-out injection schemes. We know rather accurately other parameters that determine the longitudinal acceptance, namely rf voltage, beam energy and radiation energy loss. Therefore, it may be a natural outcome that we find a good agreement between the longitudinal acceptances from measurement and simulation.

A linear approximation has been applied to the momentum compaction factor throughout the paper. In the modified lattice, the second-order term is $5.8 \times 10^{-4}$ while the first order is $7.0 \times 10^{-4}$. These parameters result in a bucket height asymmetry of only $0.1 \%$ level, and thus a linear approximation is adequate. The effect of a higher-order momentum compaction factor is normally marginal in third-generation light sources. However, it should be included into the model when the storage ring is tuned differently, e.g., in the low-alpha mode [13]. Moreover, the first-order term is typically much smaller in next generation light sources, and the higher-order terms become important.

We explore briefly how the longitudinal acceptance is shaped by the second-order term. The acceptance is computed for three typical cases as shown in Fig. 8. Based on the modified lattice parameters, the first and second order terms are varied. The rf bucket height shows an asymmetry as the second (first) order term increases (decreases). When the second-order term becomes dominant, the phase space topology transforms into the so-called alpha bucket as seen in Fig. 8. On the other hand, the 

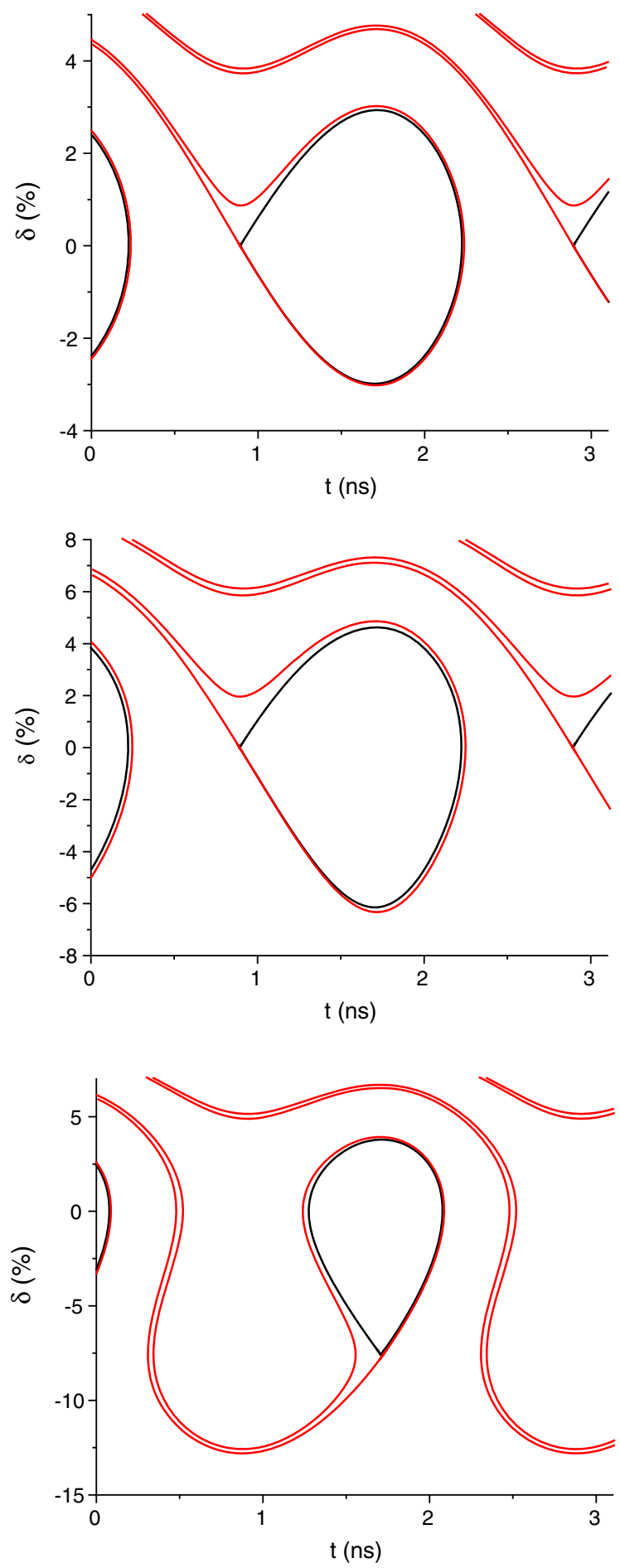

FIG. 8. Theoretical longitudinal acceptance with nonlinear momentum compaction factor. The first and second order terms are included: $\alpha$ in Eq. (1) is now $\alpha=\alpha_{1}+\alpha_{2} \delta$. Three typical cases are examined: first order dominant with $\alpha_{1}=7.0 \times 10^{-4}$ and $\alpha_{2}=5.8 \times 10^{-4}$ (top), second order predominant with $\alpha_{1}=$ $2.3 \times 10^{-4}$ and $\alpha_{2}=17.3 \times 10^{-4}$ (middle), and second order dominant with $\alpha_{1}=1.7 \times 10^{-4}$ and $\alpha_{2}=23.0 \times 10^{-4}$ (bottom). The rf voltage is set to $1.12 \mathrm{MV}$. Red lines indicate the acceptance including synchrotron radiation damping while the static bucket is indicated by black lines. longitudinal acceptance is always expanded by synchrotron radiation damping. This qualitative feature is intrinsic and not relevant to the nonlinearity of the momentum compaction factor because the damping originates in the energy dependent energy loss as we discussed in Sec. II.

\section{CONCLUSION}

The longitudinal acceptance of the BESSY II storage ring was successfully measured, and the existence of the golf-club acceptance was confirmed experimentally. To our knowledge, a measurement of this kind was performed for the first time. The experimental results show a good agreement with the prediction based on the longitudinal beam dynamics with input parameters derived from the accelerator model, including synchrotron radiation effects. As a by-product, the booster bunch length was evaluated from the measured acceptance data. The extracted bunch length is in good agreement with theoretical expectations as well.

We also examined the robustness of the momentum compaction factor against machine imperfections. It turned out that it is almost constant even when the optical functions are heavily distorted. We think that this is a generic feature at least for rings with achromat lattices. Since other relevant parameters can be known rather accurately, we conclude that the longitudinal acceptance of the electron storage ring can be predicted reliably, supporting the design of longitudinal injection schemes.

The effect of the higher-order momentum compaction factor is briefly investigated since it is important in next generation light sources. In the electron storage ring, the longitudinal acceptance is in general expanded by synchrotron radiation damping.

\section{ACKNOWLEDGMENTS}

This project has received funding from the European Union's Horizon 2020 Research and Innovation program under Grant Agreement No. 730871. We are grateful for the support and encouragement of R. Bartolini (Diamond Light Source) and Y. Papaphilippou (CERN). We would like to thank Thomas Schietinger (PSI) for proofreading the manuscript.

[1] H. Ohkuma, Top-up operation in light sources, in Proceedings of the 11th European Particle Accelerator Conference, Genoa, 2008 (EPS-AG, Genoa, Italy, 2008), pp. $36-40$.

[2] M. Aiba, M. Böge, Á. S. Hernández, F. Marcellini, and A. Streun, Longitudinal injection scheme using short pulse kicker for small aperture electron storage rings, Phys. Rev. Accel. Beams 18, 020701 (2015).

[3] B. C. Jiang, Z. T. Zhao, S. Q. Tian, M. Z. Zhang, and Q. L. Zhang, Using a double-frequency rf system to facilitate 
on-axis beam accumulation in a storage ring, Nucl. Instrum. Methods Phys. Res., Sect. A 814, 1 (2016).

[4] G. Xu, J. Chen, Z. Duan, and J. Qiu, On-axis beam accumulation enabled by phase adjustment of a doublefrequency rf system for diffraction-limited storage rings, in Proc. 7th Int. Particle Accelerator Conf. (IPAC16), pp. 2032-2035, 2016.

[5] G. Xu, Longitudinal accumulation in triple rf systems, presented at Topical Workshop on Injection and Injection System, 2017, https://indico.cern.ch/event/ 635514/overview.

[6] M.-A. Tordeux, Longitudinal injection into low-emittance ring: A novel scheme for SOLEIL upgrade, presented at Topical Workshop on Injection and Injection System, 2017, https://indico.cern.ch/event/635514/overview.

[7] BESSY II https://www.helmholtz-berlin.de/forschung/ quellen/bessy/index_en.html.

[8] M. Borland, elegant: A flexible SDDS-compliant code for accelerator simulation, Advanced Photon Source Report No. LS-287, 2000.
[9] A. Wolski, Low-emittance storage rings, Proc. of CERN Accelerator School, CERN Report No. CERN-2014-009, pp. 245-294, 2014.

[10] K. Wille, The Physics of Particle Accelerators (Oxford University Press, New York, 2000), ISBN 0-19-850549-3.

[11] P. M. Lapostolle, Proton linear accelerators: A theoretical and historical introduction, Los Alamos National Laboratory Report No. LA-11601-MS, 1989.

[12] O. Dressler and J. Feikes, Operation experiences with the BESSY II injection system, in Proceedings of the 18th Particle Accelerator Conference, New York, 1999 (IEEE, New York, 1999), pp. 1279-1281.

[13] J. Feikes, K. Holldack, P. Kuske, and G. Wüstefeld, Compressed electron bunches for $\mathrm{THz}$ generation-Operating BESSY II in a dedicated low alpha mode, in Proceedings of the 9th European Particle Accelerator Conference, Lucerne, 2004 (EPS-AG, Lucerne, 2004), pp. 2290-2292 [http://accelconf.web.cern.ch/AccelConf/ e04/]. 